\section{ARTÍCULO CORTO DE INVESTIGACIÓN}

DOI: 10.47864/SE(50)2020p1-12_121
Sociedad Colombiana de la Ciencia del Suelo

\title{
METALES EN SUELOS PRODUCTORES DE ARROZ DEL DISTRITO JUNCAL, HUILA - COLOMBIA
}

\author{
Armando Torrente Trujillo ${ }^{1} \bigotimes$ Liceth Viviana Calderón Manchola ${ }^{2}$ y Edna Milena Joven Santofimio ${ }^{2}$
}

\begin{abstract}
1 Doctor, Facultad de Ingeniería. Universidad Surcolombiana. Neiva (Huila), Colombia. e-mail:

armando.torrente@usco.ed u.co

2 M.Sc en Ingeniería y Gestión Ambiental. Facultad de Ingeniería. Universidad

Surcolombiana. Neiva (Huila), Colombia.
\end{abstract}

Palabras Claves:

Metales pesados, sistema suelo-arroz, aplicación agroquímicos, contaminación suelos de

\section{RESUMEN}

Los metales pesados como $\mathrm{Cd}, \mathrm{Cr}, \mathrm{Pb}, \mathrm{Mn}, \mathrm{Cu}$ y $\mathrm{Zn}$ procedente de los productos agroquímicos aplicados en el control de arvenses y plagas son contaminantes comunes del suelo bajo cultivo de arroz. Ciertos metales pesados en suelos agrícolas en baja concentración son esenciales para el crecimiento de las plantas ( $\mathrm{Mn}, \mathrm{Cu}$ y $\mathrm{Zn}$ ), pero algunos metales son altamente tóxicos para los humanos. Estos metales pesados tóxicos pueden ser absorbidos y acumulados por las plantas y eventualmente ingresar al cuerpo humano a través de la ingesta de alimentos. El tema ambiental como de la seguridad alimentaria relacionada con el arroz y los metales pesados ha traído una atención sustancial recientemente debido a su incidencia sobre los recursos naturales y la población que vive en áreas expuestas a contaminación por metales pesados. Para evaluar la concentración de algunos metales en suelos, se seleccionaron nueve fincas dedicadas a la producción intensiva de arroz, y se analizaron algunas propiedades físicas y químicas del suelo mediante técnicas y métodos estandarizados; la concentración de los metales se midió con espectroscopia de absorción atómica utilizando la metodología EPA 3050B. La concentración de los metales pesados analizados resultó en el rango normal a excepción del elemento Cadmio que resultó superior a 1,1 mg/kg en 6 de los 9 predios, y el elemento manganeso en la finca la Pista, donde opera una base de avionetas dedicada a la fumigación de agroquímicos.

\section{METALS IN RICE PRODUCING SOILS OF THE JUNCAL DISTRICT, HUILA - COLOMBIA}

\section{Keywords:}

Heavy metals, soilrice system, agrochemical application, contamination

\section{ABSTRACT}

Heavy metals such as $\mathrm{Cd}, \mathrm{Cr}, \mathrm{Pb}, \mathrm{Mn}, \mathrm{Cu}$ and $\mathrm{Zn}$ from agrochemical products applied in the control of weeds and pests are common contaminants of the soil under rice cultivation. Certain heavy metals in low concentration agricultural soils are essential for plant growth ( $\mathrm{Mn}, \mathrm{Cu}$ and $\mathrm{Zn}$ ), but some metals are highly toxic to humans. These toxic heavy metals can be absorbed and accumulated by plants and eventually enter the human body through food intake. The environmental issue such as food security related to rice and heavy metals has attracted substantial attention recently due to its impact on natural resources and the population living in areas exposed to heavy metal contamination. To evaluate the concentration of some metals in soils, nine farms dedicated to intensive rice production were selected, and some physical and chemical properties of the soil were analyzed using standardized techniques and methods; the concentration of metals was measured with atomic absorption spectroscopy using the EPA 3050B methodology. The concentration of heavy metals analyzed was in the normal range, except for the element Cadmium, which was higher than $1.1 \mathrm{mg}$ / $\mathrm{kg}$ in 6 of the 9 farms, and the element manganese in the La Pista farm, where a light aircraft base operates. dedicated to the fumigation of agrochemicals 


\section{INTRODUCCIÓN}

El arroz (Oryza sativa) es un alimento básico que se utiliza para el consumo diario y proporciona más del $70 \%$ de la energía derivada de la ingesta diaria de alimentos, además ocupa el primer lugar en términos de valor económico entre los cultivos de ciclo corto y es el tercer producto agrícola en extensión cultivada después del café y el maíz en Colombia (Dane Fedearroz 2020). El arroz es la principal fuente de exposición a metales pesados por el paquete tecnológico en el control de arvenses, plagas y enfermedades que se aplica en la práctica intensiva de este monocultivo en el sur del Tolima y el norte del Huila, región con el mayor rendimiento promedio de 7,4 ton/ha en producción de arroz bajo riego superficial (Torrente 2018). El consumo de arroz contaminado con metales pesados está estrechamente relacionado con el impacto en la salud y al ambiente.

En Colombia, existen diversos estudios sobre la contaminación en el agua por sustancias químicas que puedan incidir en la salud (Espinosa 2018, Velásquez 2017, Gualdron 2016, Tobón et al 2010); no obstante, la información sobre la contaminación en suelos es escasa y carece de los criterios y estándares de calidad. Es indispensable la gestión de entidades del estado con el fin de desarrollar investigación en diferentes sectores agrícolas primarios, para contribuir a garantizar la producción agrícola y la sostenibilidad ambiental del recurso suelo (Rueda et al 2011).

Tradicionalmente el suelo ha sido considerado como un depositario de cantidades limitadas de desechos humanos y animales, debido a su capacidad de regeneración. Sin embargo, la contaminación en el suelo es uno de los procesos que junto con la erosión contribuye a la rápida degradación del mismo, a la debilitación de su fertilidad y acumulación de sustancias que inhiben el crecimiento de las plantas, siendo las principales consecuencias de su desgaste químico. Sin lugar a duda las explotaciones mineras, la contaminación del agua, las plantas y animales por cuenta de la industrialización, los fertilizantes, insecticidas químicos y otras actividades propias del desarrollo han propiciado el incremento de metales pesados en el suelo. Como consecuencia, estos elementos potencialmente tóxicos se acumulan lentamente en el perfil del suelo, influyendo en el equilibrio natural de las funciones del suelo y disminuyendo su capacidad de producción y amortiguación de contaminantes (Nicholson et al 2003). Demostrar que un suelo se encuentra contaminado, no es una tarea fácil, y más si se trata de metales pesados, puestos que pueden ser encontrados en el ambiente de forma natural o por factores antrópicos (Brizuela y Jiménez 2012).

Sin duda alguna, el suelo es uno de los recursos más importantes para garantizar la alimentación del hombre y de los amínales, es una composición de elementos minerales y orgánicos producto de procesos de meteorización que a su vez conllevan cambios físico, químicos y biológicos, lo que genera unas características particulares que están asociadas al ecosistema en donde se desarrolle. El estudio de las características morfológicas, así como de las propiedades químicas y físicas de los suelos es fundamental, ya que permite conocer el nivel de degradación y afectación de indicadores que se relacionan con la fertilidad y tener una comprensión más definida de las respuestas de los cultivos a la aplicación de fertilizantes para obtener los mayores rendimientos (Bernal et al 2019).

El uso de agroquímicos genera impactos para la salud. La precarización laboral y la insuficiente capacitación para el manejo de agroquímicos coloca a los trabajadores rurales como una de las principales poblaciones de riesgo; a pesar de que la reglamentación establece distintas limitaciones para el manejo de agroquímicos, existen diferentes problemáticas en la aplicación de la normativa y en su control; y otro aspecto fundamental es que la población en general desconoce los riesgos para la salud y el ambiente emergentes de la tecnificación de la producción y de las sustancias que se utilizan.

Los metales pesados representan algunos de los contaminantes más tóxicos para el medio ambiente, debido a su naturaleza no biodegradable, alta solubilidad y su potencialidad para acumularse en diferentes partes del cuerpo; éstos tienen efectos secundarios y graves para la salud humana y 
representan también un riesgo ambiental (Heinzen \& Rodríguez 2016, Londoño et al 2016). Es importante mencionar que los metales se encuentran de manera natural en el medio ambiente, en concentraciones que por lo general, no afectan las diversas formas de vida (Reyes et al 2016).

Los metales tóxicos como el $\mathrm{Cd}$, $\mathrm{As}$ y $\mathrm{Pb}$, se encuentran en los suelos cultivados en arroz de algunas provincias de China. Generalmente, los estudios sobre la afectación al suelo y al ambiente de los elementos tóxicos aplicados al arroz son limitados y se centran en un solo elemento o en sitios específicos de muestreo (Fanfu Zeng et al 2015, Li 2015). Los metales pesados cancerígenos como $\mathrm{Cd}$, $\mathrm{Cr}$, As y $\mathrm{Ni}$ y aquellos con potencial toxicidad para el sistema nervioso como $\mathrm{Mn}, \mathrm{Hg}$ y $\mathrm{Pb}$, no se han analizado sistemáticamente $\mathrm{y}$ es escasa la información sobre factores de riesgo a la salud de estos elementos (Yuang et al 2014).

Estudios demuestran la existencia importante de Arsénico (As), Cadmio (Cd), Cobre (Cu), Cobalto (Co), Zinc ( $\mathrm{Zn})$, Hierro (Fe), Manganeso (Mn), Níquel (Ni) y Plomo ( $\mathrm{Pb}$ ) en los plaguicidas normalmente utilizados en cultivos como el arroz (Díaz et al 2018, Trujillo 2005, Boluda et al 2004). No obstante, en Colombia no se cuenta con un marco legal y normativo que indique los niveles máximos permisibles para metales pesados en suelos y que regule los procesos de producción de arroz en el país, lo cual inhibe el control sobre la aplicación de agroquímicos. Mahecha et al 2017, recopilaron estudios sobre metales pesados en sistemas de producción agrícola para establecer una línea base que permitiera identificar necesidades futuras de investigación en esta temática. Entre los resultados se identificó que los metales pesados estudiados en el país son $\mathrm{Cd}>\mathrm{Pb}>\mathrm{Hg}>\mathrm{Cr}>\mathrm{Ni}>\mathrm{Cu}=\mathrm{Zn}=\mathrm{As}>\mathrm{Mn}>\mathrm{Fe}$, destacándose el $\mathrm{Cd}, \mathrm{Pb}, \mathrm{Hg}$ y $\mathrm{Cr}$. Se resalta la importancia de generar investigaciones en valores de referencia para estos elementos en los suelos colombianos, que permitan establecer programas de monitoreo y de evaluación en posibles casos de contaminación (Rueda et al 2011).

Por lo anterior, se establece como objetivo evaluar la incidencia del Plomo $(\mathrm{Pb})$, Cadmio (Cd), Cromo $(\mathrm{Cr})$, Manganeso $(\mathrm{Mn})$, Cobre (Cu) y Zinc $(\mathrm{Zn})$ en suelos dedicados a la producción intensiva de arroz en el área del Juncal en el departamento del Huila, teniendo en cuenta que este departamento es uno de los principales productores de arroz del país. En los inventarios de Torrente (2018) en suelos del Huila, reconoce un promedio de 20 productos agroquímicos o plaguicidas utilizados por campaña de arroz y constituidos especialmente por herbicidas, funguicidas, insecticidas, que utilizan como ingredientes activos sustancias de la familia organoclorados, organofosforados, piretroides, carbamatos y otros compuestos plaguicidas.

\section{Materiales y métodos}

\section{Ubicación geográfica del área}

El distrito el Juncal se ubica en la jurisdicción del mismo nombre en el área rural del municipio de Palermo-Huila (N 2'51'54” - W 75'19'30”), es una zona apta para la producción agrícola y pecuaria; su principal fuente hídrica es la laguna el Juncal, el cual es alimentada por el río Magdalena mediante un sistema de bombeo a través de un canal aductor cuya función es irrigar aproximadamente 3397 has con predominio en producción de arroz bajo riego superficial en clima cálido seco. La franja altitudinal es de 500 a $700 \mathrm{msnm}$, temperatura promedio diaria mayor a $24^{\circ} \mathrm{C}$ y precipitación promedia anual de $1380 \mathrm{~mm}$, allí se identificaron 3 grupos de mapeo del suelo (figura 1). 

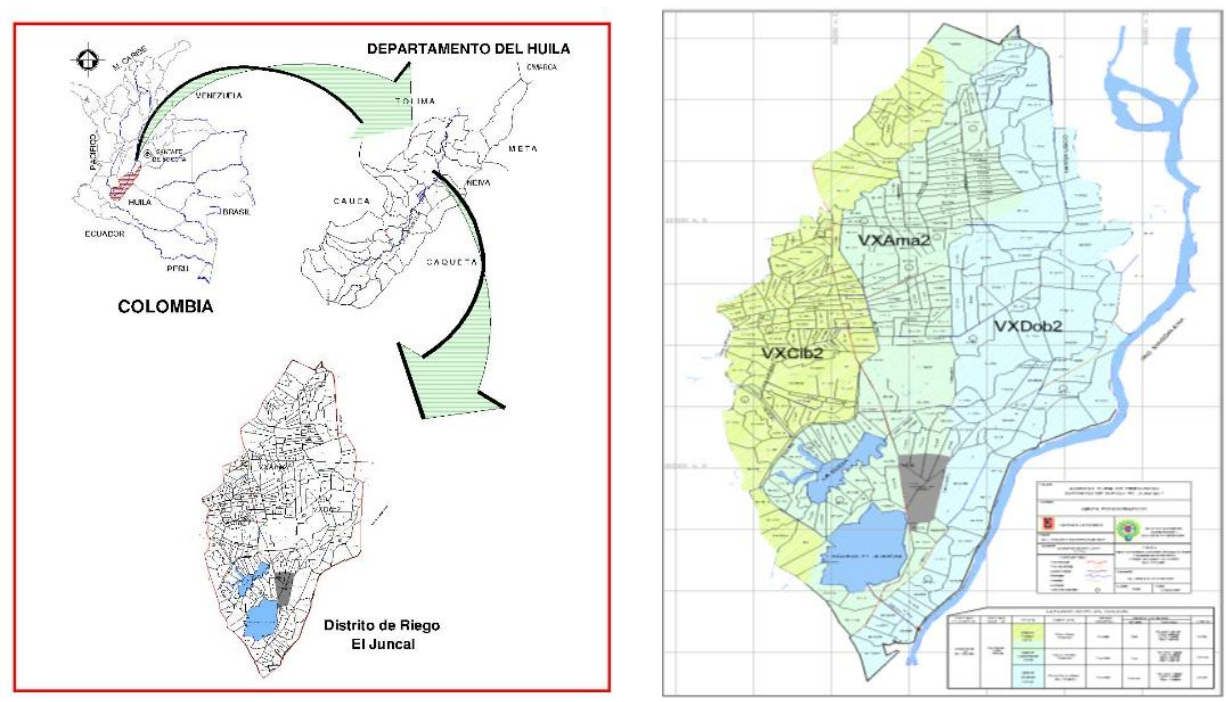

Figura 1. Localización del Distrito el Juncal y unidades de mapeo del suelo

\section{Recolección y técnica de análisis de suelos}

La recolección de las muestras se realizó en dos periodos diferentes (periodo seco y periodo húmedo en el año) cubriendo el paisaje fisiográfico del Distrito de Riego el Juncal en las diferentes posiciones así: terraza antigua, terraza subreciente y terraza reciente (figura 2).

\begin{tabular}{|c|c|c|c|c|c|c|c|}
\hline \multicolumn{8}{|c|}{ LEYENDA MAPA DE SUELOS } \\
\hline $\begin{array}{l}\text { PROVINCMA } \\
\text { FISIOGRAAICA }\end{array}$ & $\begin{array}{l}\text { PROVINCIA } \\
\text { CLIMÁTICA }\end{array}$ & PAISA.JE & SUB-PAISA.JE & $\begin{array}{l}\text { UNIDAD } \\
\text { DEMAPEO }\end{array}$ & \multicolumn{2}{|c|}{ UNIDAD TAXONOMICA } & SIMBOLO \\
\hline \multirow{3}{*}{$\begin{array}{l}\text { Planicie Aluvial } \\
\text { Rio diagdalena }\end{array}$} & \multirow{3}{*}{$\begin{array}{l}\text { Piso Témimoo } \\
\text { Calidion } \\
<1000 \text { nts. }\end{array}$} & $\begin{array}{l}\text { TERRAZA } \\
\text { ANIIGLAa } \\
\text { VXA1a }\end{array}$ & $\begin{array}{l}\text { Plano Concava } \\
\text { Erosionada }\end{array}$ & Complejo & Cielo & $\begin{array}{l}\text { Fluventic Haplustoll } \\
\text { Accuic Ustifluevent } \\
\text { Acuic ustopept } \\
\text { Tipyc Haplustaff }\end{array}$ & $\mathrm{vxcib2}$ \\
\hline & & $\begin{array}{l}\text { TERRAZA } \\
\text { SUB-RECIENTE } \\
\text { VXCila }\end{array}$ & $\begin{array}{l}\text { Concava-Convexa } \\
\text { Erosionada }\end{array}$ & Asociacion & Ama & $\begin{array}{l}\text { Fluventic Ustropept } \\
\text { Ustic Dostropept } \\
\text { Tipyc Haplustaf } \\
\text { Tipyc Ustifluvent }\end{array}$ & VXAma2 \\
\hline & & $\begin{array}{l}\text { TERRAZA } \\
\text { RECIENTE } \\
\text { VXDo1a }\end{array}$ & $\begin{array}{l}\text { Plano-ConcavaCorvexa } \\
\text { Mod. Erosionada }\end{array}$ & Asociacion & Domador & $\begin{array}{l}\text { Fluventic Ustropept } \\
\text { Lithic Utstorhert } \\
\text { Tilipyc Ustorthent }\end{array}$ & VXDob2 \\
\hline
\end{tabular}

Figura 2. Descriptivo fisiográfico y leyenda mapa de suelos

Se realizó el muestreo de suelos por el método sistemático en zigzag, mediante la recolección de 15 submuestras por finca seleccionada para la investigación, tomadas a $30 \mathrm{~cm}$ de profundidad. El laboratorio donde se procesaron las muestras para los análisis de metales en suelos certificó la acreditación ante el IDEAM y un sistema de gestión basado en la NTC-ISO/IEC 17025. Las metodologías para la caracterización de los suelos fueron las siguientes: color con tabla Munsell, textura por Bouyoucos, densidad aparente por el cilindro de volumen conocido, densidad real por el picnómetro, carbono orgánico por Walkley - Black, metales por absorción atómica EPA 3050B, pH potenciométrico relación agua- suelo 1:1 y CIC por acetato de amonio a $\mathrm{pH} 7,1 \mathrm{~N}$. 
Previo a la determinación de los parámetros físicos y químicos del suelo, se realizó pre-tratamiento a las muestras recolectadas con el fin de asegurar resultados correctos y de seguir los protocolos establecidos por las técnicas de análisis. Por lo tanto se sometieron a los siguientes procesos: secado, trituración, tamizado, cuarteo y análisis. Simultáneamente se realizó la búsqueda de normatividad internacional de niveles máximos permisibles de metales pesados en suelos para agricultura, con el fin de establecer si efectivamente estos excedían el nivel máximo permisible.

Lo análisis se realizaron por triplicado. En todos los procedimientos se emplearon blancos, para verificar la ausencia de contaminación de las muestras durante el procedimiento analítico y los reactivos empleados fueron de calidad analítica adecuada para este tipo de estudio.

Todos los blancos presentaron niveles de los analitos de interés por debajo de los límites de detección de los métodos, lo que indica ausencia de contaminación de las muestras durante las etapas de muestreo, almacenamiento, transporte al laboratorio y preparación de las muestras. Los resultados demuestran que los procedimientos empleados para la determinación de los metales en las matrices de interés brindan resultados veraces y precisos, y por tanto, los mismos pueden emplearse para la determinación de dichos metales en muestras colectadas.

\section{RESULTADOS}

Los suelos característicos del área del Juncal son de mediana a fuertemente ácidos en el perfil estratigráfico con contenidos bajos de materia orgánica y baja capacidad de intercambio iónico con excepciones en el horizonte superficial, ausencia de fósforo aprovechable, alta concentración de aluminio y alta saturación de bases excepto en el horizonte superficial. La alta concentración de aluminio es la responsable de la fuerte acidez intercambiable del suelo. En cuanto a elementos menores son características las deficiencias de manganeso, cinc y boro, nutrientes esenciales para el desarrollo de las plantas.

El perfil de suelo es de textura fina a media, de bloques angulares a masivos, bien drenados, con disponibilidad media de agua aprovechable para las plantas. El nivel freático se halla muy profundo (> $8 \mathrm{~m}$ ). Los horizontes del suelo presentan cutanes de arcilla y manchas pardas amarillentas pálidas en todo el perfil del suelo. En la constitución de la matriz del material parental dominan las arcillolitas cuya consistencia en húmedo es extremadamente firme.

La fracción arena está dominada por cuarzo (94\%), con inclusiones de hematita (3\%), feldespatos (2\%) y fitolitos (1\%). En la fracción arcilla domina la caolinita (78\%), que incluye esmectitas (8\%). El mineral dominante es el cuarzo muy resistente a la meteorización y como consecuencia ofrece escaso aporte de elementos potencialmente disponibles para los procesos de nutrición vegetal. El cuarzo se presenta en granos de tamaño variado, irregulares y subredondeados principalmente. Algunos granos tienen manchas con óxidos de hierro. La hematita son nódulos irregulares subredondeados y los feldespatos plagioclasas están en proceso de caolinización, ligeramente alterados (Torrente, 2012).

Los suelos se ubican sobre la margen izquierda de la planicie aluvial de la Cuenca Alta del Río Magdalena en posiciones de terraza baja, media y alta con relieve plano a ligeramente plano, siendo de origen sedimentario, desarrollado en régimen de humedad ústico y clima edáfico isohipertérmico con formación de plintita. Estas planicies son utilizadas para la siembra intensiva de cultivo de arroz bajo riego por el método de melgas en contorno (curvas de nivel).

\section{Análisis físicos de suelos}

En la tabla 1 se observan los resultados de los análisis físicos del suelo en las nueve fincas seleccionadas para el estudio en el Distrito El Juncal. 
Tabla 1. Análisis físico de los suelos

\begin{tabular}{|l|c|c|c|c|c|c|c|c|}
\hline Finca & $\begin{array}{c}\text { Materia } \\
\text { orgánica } \\
(\%)\end{array}$ & $\begin{array}{c}\text { Arena } \\
(\%)\end{array}$ & $\begin{array}{c}\text { Limo } \\
(\%)\end{array}$ & $\begin{array}{c}\text { Arcilla } \\
(\%)\end{array}$ & Textura & $\begin{array}{c}\text { Densidad } \\
\text { aparente } \\
\text { g.cm }^{-3}\end{array}$ & $\begin{array}{c}\text { Densidad } \\
\text { real } \\
\text { g.cm } \text { - }^{-3}\end{array}$ & $\begin{array}{c}\text { Color en } \\
\text { seco }\end{array}$ \\
\hline Villa Milena & 1,28 & 56 & 24 & 20 & FArA & 1,48 & 2,55 & 10 YR1/2 \\
\hline La Laguna & 2,20 & 48 & 26 & 26 & FArA & 1,43 & 2,53 & 10 YR3/3 \\
\hline El Silencio & 2,43 & 28 & 22 & 50 & Ar & 1,39 & 2,48 & 10 YR3/4 \\
\hline Mi Finquita & 1,06 & 50 & 23 & 27 & FArA & 1,52 & 2,50 & 10 YR5/4 \\
\hline La Pista & 3,03 & 52 & 18 & 30 & FArA & 1,50 & 2,52 & 10 YR2/2 \\
\hline El Lago & 1,10 & 44 & 22 & 34 & FAr & 1,51 & 2,53 & 10 YR4/3 \\
\hline Yuventus & 3,01 & 40 & 20 & 40 & FAr & 1,42 & 2,52 & 10 YR2/2 \\
\hline Alejandra & 1,43 & 70 & 8 & 22 & FArA & 1,51 & 2,58 & 10 YR3/3 \\
\hline Sinaí & 1,53 & 62 & 12 & 26 & FArA & 1,43 & 2,53 & 10 YR3/3 \\
\hline
\end{tabular}

FArA Franco arcillo arenoso, FAr Franco arcilloso, Ar arcilloso

Los suelos proceden de los aluviones del rio Magdalena y sus afluentes, su textura dominante es franco arcillo arenosa con densidad aparente y densidad real

\section{Análisis químico de suelos}

El análisis de Cadmio (Cd), Cromo $(\mathrm{Cr})$, Plomo $(\mathrm{Pb})$, Manganeso $(\mathrm{Mn})$, Cobre $(\mathrm{Cu})$ y Zinc $(\mathrm{Zn})$, permitieron el reconocimiento de la concentración de estos metales en suelos con cultivo de arroz y verificar sus niveles frente a normas internacionales. Además se determinó la capacidad de intercambio catiónico y el pH del suelo.

Los suelos presentan baja capacidad de intercambio catiónico (CIC) a excepción de Yuventus, El Silencio característicos de suelos minerales intervenidos con bajos contenidos de materia orgánica y notación de color pardo amarillento por sus altos contenidos en óxidos de hierro.

y La Pista cuya CIC es moderada, lo que correlaciona con los mayores contenidos de materia orgánica y grupos texturales con moderado a alto contenido de arcilla (30 a 50\%) en el suelo. Los suelos muestran $\mathrm{pH}$ de medianamente acido a muy fuertemente acido características que favorecen la disponibilidad de los elementos esenciales menores en la nutrición vegetal como son manganeso, cobre y cinc. El carácter acido de los suelos está asociado al manejo con prácticas agrícolas intensivas en el monocultivo de arroz con excesivas láminas de riego y bajas eficiencias por concepto de escorrentía y percolación (tabla 2).

Tabla 2. Análisis químico de los suelos

\begin{tabular}{|l|c|c|c|c|c|c|c|c|}
\hline \multirow{2}{*}{ Finca } & \multicolumn{9}{|c|}{$\mathrm{mg}^{-1}$} & \multicolumn{2}{c|}{$\mathrm{Cmol}^{-1} \mathrm{~kg}^{-1}$} & \multirow{2}{*}{$\mathrm{pH}$} \\
\cline { 2 - 11 } & $\mathrm{Cd}$ & $\mathrm{Cr}$ & $\mathrm{Pb}$ & $\mathrm{Mn}$ & $\mathrm{Cu}$ & $\mathrm{Zn}$ & $\mathrm{CIC}$ & \\
\hline Villa Milena & $<0,6$ & $<18$ & 28 & 475 & 12 & 38 & 6,03 & 4,68 \\
\hline La Laguna & $<0,6$ & $<18$ & 25 & 58 & 10 & 21 & 9,25 & 6,05 \\
\hline El Silencio & 2,4 & 23 & 33 & 127 & 15 & 77 & 13,59 & 4,78 \\
\hline Mi Finquita & 2,6 & $<18$ & 27 & 385 & 13 & 27 & 7,03 & 5,62 \\
\hline La Pista & 2,4 & 62 & 38 & 1452 & 24 & 77 & 17,36 & 5,36 \\
\hline El Lago & 2,2 & $<18$ & 20 & 396 & 19 & 54 & 6,03 & 4,81 \\
\hline Yuventus & 2,0 & $<18$ & 28 & 39 & 14 & 29 & 12,73 & 5,22 \\
\hline Alejandra & 2,8 & 29 & 33 & 84 & 17 & 48 & 6,84 & 4,47 \\
\hline Sinaí & $<0,6$ & $<18$ & 37 & 304 & 17 & 31 & 5,22 & 4,84 \\
\hline
\end{tabular}


Los suelos son medianos a fuertemente ácidos con efectos por presencia de Aluminio (Al), se infiere deficiencia de $\mathrm{Ca}, \mathrm{K}, \mathrm{N}, \mathrm{Mg}$, Mo, P y S y se observa una actividad microbiana escasa. Los valores de $\mathrm{pH}$ son el resultado de las altas láminas de riego utilizadas en el cultivo de arroz, que finalmente producen un lavado de las bases del suelo y su intercambio con radicales acídicos como hidrogeno y aluminio. Es importante tener en cuenta que estos valores de $\mathrm{pH}$, no son constantes y varían acorde con los procesos de saturación e inundación del suelo, los procesos de óxido-reducción y principalmente por los aportes nitrogenados en algunas épocas del año.

Los elementos manganeso $(\mathrm{Mn})$, cobre $(\mathrm{Cu})$ y cinc $(\mathrm{Zn})$ hacen parte de los microelementos nutrientes esenciales para las plantas, en cambio los elementos cadmio $(\mathrm{Cd})$, cromo $(\mathrm{Cr})$ y plomo $(\mathrm{Pb})$ son metales pesados tóxicos en el suelo cuyo problema radica en la semejanza entre algunos de estos contaminantes y los elementos trazas esenciales, las células pueden incorporar los elementos tóxicos del suelo a sus membranas, causando un daño letal cuando ingresan a los organismos consumidores.

Los microlementos juegan un papel importante en el metabolismo y en un gran número de reacciones enzimáticas. Un exceso o ausencia puede llevar a un aumento en la susceptibilidad a enfermedades. El nivel de $\mathrm{pH}$ fuertemente acido en la zona de las raíces conlleva una alta disponibilidad de la mayoría de los microelementos. La concentración de estos microelementos esenciales en los suelos del Juncal se hallan en el rango normal (Fadigas et al 2006, Rueda et al 2011) a excepción de Mn en la Pista, cuya concentración es superior a 600 ppm, y esto se explica por las operaciones diarias de fumigación con plaguicidas en la base aérea establecida en el predio.

El nivel de manganeso encontrados son valores medios comúnmente en suelos $(600 \mathrm{mg} / \mathrm{kg})$, y en el caso de la concentración de la Pista son similares a aquellas que se consideran fitotóxicas (>1400 $\mathrm{mg} / \mathrm{kg}$ ) (Bighman y Bartels 1996). El manganeso no es reconocido como un metal que contamina los suelos, sino como un micronutriente, pero se acumula en el suelo superficial y puede causar efectos tóxicos en algunas plantas. En La Pista con los niveles más altos de Mn (tabla 2), son suelos alterados por la incidencia intensiva de agroquímicos dedicados al cultivo de arroz.
Los metales pesados analizados en los suelos del Juncal $(\mathrm{Cd}, \mathrm{Cr}$ y $\mathrm{Pb})$ se clasifican en los rangos normales a excepción de cromo en La Pista, y cadmio en El Silencio, Mi Finquita, La Pista, El Lago, Yuventus y Alejandra, cuya concentración resultó igual o superior a $2 \mathrm{ppm}$. Se obtuvieron concentraciones de $\mathrm{Cd}$ superiores a los establecidos por las legislaciones $\mathrm{y}$ reglamentaciones, en suelos agrícolas (Fadigas et al 2006, Rueda et al 2011).

La alta concentración de Cadmio en el suelo influye en la actividad de los procesos orgánicos del suelo, este elemento permanece inmóvil en el suelo y puede ser incorporado por plantas, entrando así a la cadena alimentaria (ATSDR). El cadmio presenta baja afinidad por formas adsorbentes, lo que repercute en alta solubilidad y movilidad, produciendo efectos altamente tóxicos en suelos, plantas, microorganismos y seres humanos. En forma natural, el cadmio está asociado con minerales de fósforo y zinc, por esta razón se puede encontrar en fertilizantes o llegar a convertirse en metal bioacumulable a partir de suelos con aplicación continuada de fertilizantes fosfatados o abonos orgánicos (Bravo et al. 2013. Charrupi y Martínez 2017).

Se ha encontrado que el Cadmio desencadena una serie de reacciones metabólicas que promueven un gran número de cambios en las plantas, como lo es inducirla a diferentes tipos de expresión génica e incrementa la actividad de enzimas antioxidantes como por ejemplo las peroxidasas (III) y las súper oxido dismutasa (SOD), las cuales les ayudan a hacer frente al estrés oxidativo ocasionado por los radicales libres, ayudando a prolongar su vida. Sin embargo, una acumulación de cadmio en exceso, conlleva a cambios morfológicos, estructurales, fisiológicos, bioquímicos y moleculares como lo son la desorganización de cloroplastos (Alterando la tasa fotosintética), cambios en el número de granos y deformación en el núcleo de las células de la raíz (Castro et al 2015).

Los valores de Cromo (Cr) son los de menor exactitud analítica, ya que la técnica empleada por el laboratorio, presentó un límite de cuantificación de 18 ppm. El manganeso $(\mathrm{Mn})$ presenta alta concentración en la Finca La Pista, siendo perjudicial para el normal desarrollo del cultivo. Este nivel puede generar toxicidad en las plantas e influye negativamente sobre el crecimiento de las mismas. Una acidificación excesiva del suelo, puede 
producir una gran liberación de manganeso, puesto que aumenta su solubilidad en la solución del suelo (Sierra 2019).

A pesar de que el plomo es un elemento muy tóxico, su movilidad es muy baja en el suelo, incluso en los de bajo $\mathrm{pH}$ como los de la zona de estudio. En los suelos, este elemento puede precipitar como hidróxido, fosfato o carbonato, que son compuestos altamente estables y poco propicios de pasar a las plantas. También es posible la formación de complejos orgánicos muy estables que disminuyen la solubilidad del metal.

\section{Análisis estadístico de suelos}

Las estadísticas de los parámetros físicos de los suelos estudiados denotan la dominancia de la fracción arena y el bajo contenido de materia orgánica en el suelo, lo que determina una menor relación de intercambio y almacenamiento de agua y nutrientes en el suelo (tabla 3).

Tabla 3. Algunas características físicas de los suelos del Juncal

\begin{tabular}{|l|l|l|l|l|l|l|l|}
\hline Parámetro & $\mathrm{N}$ & Mín. & Máx. & $\overline{\boldsymbol{x}}$ & $\mathrm{S}$ & $\mathrm{S}^{2}$ & $\mathrm{CV}$ \\
\hline Materia orgánica (\%) & 9 & 1,06 & 3,03 & 1,90 & 0,79 & 0,621 & 0,42 \\
\hline Arena (\%) & 9 & 28 & 70 & 50 & 12,29 & 151 & 0,25 \\
\hline Limo (\%) & 9 & 8 & 26 & 19,44 & 5,90 & 34,778 & 0,30 \\
\hline Arcilla (\%) & 9 & 20 & 50 & 30,56 & 9,48 & 89,778 & 0,31 \\
\hline D. aparente $\left(\mathrm{g} . \mathrm{cm}^{-3}\right)$ & 9 & 1,39 & 1,52 & 1,47 & 0,05 & 0,002 & 0,03 \\
\hline D. real $\left(\mathrm{g} . \mathrm{cm}^{-3}\right)$ & 9 & 2,48 & 2,58 & 2,53 & 0,03 & 0,001 & 0,01 \\
\hline
\end{tabular}

Los metales pesados disminuyeron en el siguiente orden: $\mathrm{Mn}>\mathrm{Zn}>\mathrm{Pb}>\mathrm{Cr}>\mathrm{Cu}>\mathrm{Cd}$, presentando el Mn la mayor dispersión de valores, es decir la mayor varianza y coeficiente de variación superior al $100 \%$. La capacidad de carga iónica es baja, lo que se asocia al carácter acido de los suelos (tabla 4).

Tabla 4. Algunas características químicas de los suelos del Juncal

\begin{tabular}{|l|l|l|l|l|l|l|l|}
\hline Parámetro & N & Mín. & Máx. & $\overline{\boldsymbol{x}}$ & $\mathrm{S}$ & $\mathrm{S}^{2}$ & $\mathrm{CV}$ \\
\hline Cadmio $\left(\mathrm{mg} \cdot \mathrm{kg}^{-1}\right)$ & 9 & 0,6 & 2,8 & 1,95 & 0,87 & 0,751 & 0,44 \\
\hline Cromo $\left(\mathrm{mg} \cdot \mathrm{kg}^{-1}\right)$ & 9 & 18 & 62 & 25,50 & 15,27 & 233,14 & 0,60 \\
\hline Plomo $\left(\mathrm{mg} \cdot \mathrm{kg}^{-1}\right)$ & 9 & 20 & 38 & 30,13 & 6,20 & 38,41 & 0,21 \\
\hline Manganeso $\left(\mathrm{mg}^{-1} \mathrm{~kg}^{-1}\right)$ & 9 & 39 & 1452 & 355,63 & 466,3 & 217439,7 & 1,31 \\
\hline Cobre $\left(\mathrm{mg} \cdot \mathrm{kg}^{-1}\right)$ & 9 & 10 & 24 & 16,13 & 4,22 & 17,84 & 0,26 \\
\hline Cinc $\left(\mathrm{mg} \cdot \mathrm{kg}^{-1}\right)$ & 9 & 21 & 77 & 45,50 & 22,32 & 498,29 & 0,49 \\
\hline CIC $\left(\mathrm{Cmol} \cdot \mathrm{kg}^{-1}\right)$ & 9 & 5,2 & 17,3 & 9,76 & 4,34 & 18,86 & 0,45 \\
\hline pH & 9 & 4,47 & 6,05 & 5,14 & 0,52 & 0,27 & 0,10 \\
\hline
\end{tabular}

La tabla 5 muestra la correlación existente entre los distintos elementos, con asterisco (*) se identifican las correlaciones que resultaron estadísticamente significativas $(\mathrm{p}<0.05)$. Se encontraron las correlaciones de $\mathrm{Cd}-\mathrm{Zn}, \mathrm{Cr}-(\mathrm{Pb}, \mathrm{Mn}, \mathrm{Cu}, \mathrm{Zn}$ y CIC), $\mathrm{Mn}-(\mathrm{Cu}, \mathrm{Zn}), \mathrm{Cu}-\mathrm{Zn}$ y, Zn - CIC. El Cr correlacionó con todos los demás elementos analizados y la CIC; el Cd correlacionó solo con el Zn y el Mn correlacionó con $\mathrm{Cu}$ y $\mathrm{Zn}$, a su vez $\mathrm{Cu}$ correlacionó con $\mathrm{Zn}$. Las correlaciones identificadas pudieran indicar fuentes comunes en los suelos para estos elementos. Para el $\mathrm{Pb}$ no se encontró correlación significativa con los demás elementos, lo que sugiere que la fuente de este elemento en los suelos pudiera ser de distinto origen que para el resto de los metales (tabla 5). 
Tabla 5. Correlación lineal Pearson

\begin{tabular}{|l|l|l|l|l|l|l|l|}
\hline PARAMETRO & $\mathrm{Cr}$ & $\mathrm{Pb}$ & $\mathrm{Mn}$ & $\mathrm{Cu}$ & $\mathrm{Zn}$ & $\mathrm{CIC}$ & $\mathrm{pH}$ \\
\hline $\mathrm{Cd}$ & 0,376 & 0,065 & 0,154 & 0,464 & $0,522^{*}$ & 0,361 & $-0,176$ \\
\hline $\mathrm{Cr}$ & - & $0,600^{*}$ & $0,852^{*}$ & $0,779^{*}$ & $0,664^{*}$ & $0,703^{*}$ & 0,057 \\
\hline $\mathrm{Pb}$ & & - & 0,406 & 0,469 & 0,400 & 0,408 & $-0,227$ \\
\hline $\mathrm{Mn}$ & & & - & $0,729^{*}$ & $0,528^{*}$ & 0,475 & 0,099 \\
\hline $\mathrm{Cu}$ & & & & - & $0,706^{*}$ & 0,403 & $-0,305$ \\
\hline $\mathrm{Zn}$ & & & & & - & $0,596^{*}$ & $-0,396$ \\
\hline $\mathrm{CIC}$ & & & & & & - & 0,279 \\
\hline
\end{tabular}

*Correlaciones significativas para $\mathrm{p}<0,05$

Debido a la semejanza química del $\mathrm{Cd}$ y $\mathrm{Zn}$, su interacción ha sido la más estudiada. Se han descrito efectos entre ambos elementos que han causado algunas controversias al respecto. Debido a esta situación, no se ha podido hacer una generalización; aunque McBride 1995, observó que el incremento en las cantidades de Zn en el suelo, tiene efectos inhibitorios sobre la absorción de $\mathrm{Cd}$ y viceversa; sin embargo, la relación parece depender de la concentración de ambos elementos y de la especie vegetal expuesta. El comportamiento químico del cadmio se asemeja al del zinc, pero es mucho más afín al azufre y más móvil en ambientes ácidos. La dinámica del $\mathrm{Cd}$ está fuertemente afectada por el $\mathrm{pH}$, siendo los sesquióxidos quienes controlan la solubilidad del metal, ya que la materia orgánica es baja en estos suelos ácidos.

Debido a la importancia ambiental de la acumulación de cadmio en el suelo, se han investigado varias técnicas para el manejo de suelos ricos en cadmio. Estas técnicas se basan en la realización de lavados en suelos ácidos, o bien en el aumento del pH (por ejemplo el encalado) y la capacidad de intercambio de cationes (Estévez et al 2000).

\section{CONCLUSIONES}

Los niveles de $\mathrm{Cd}, \mathrm{Cr}, \mathrm{Pb}, \mathrm{Mn}, \mathrm{Cu}$ y $\mathrm{Zn}$ en los suelos con cultivo de arroz en el distrito de riego el Juncal en el municipio de Palermo - Huila variaron en los siguientes rangos: $\mathrm{Cd}(0.6-2.8 \mathrm{mg} / \mathrm{kg}), \mathrm{Cr}(18-62$ $\mathrm{mg} / \mathrm{kg}$ ), $\mathrm{Pb}$ (20-38 mg/kg), Mn (39-1452), Cu (10-24) y Zn (21-77 mg/kg). Las mayores concentraciones se encontraron en la Finca La Pista, con altos niveles de
Mn y Cd en el suelo, ya que son próximos a los niveles considerados como fitotóxicos y superan los límites en algunas normas internacionales.

La concentración de $\mathrm{Pb}, \mathrm{Cr}, \mathrm{Mn}, \mathrm{Cu}$ y $\mathrm{Zn}$ son normales de acuerdo con los niveles de referencia promedios en el marco legal de diferentes regiones del mundo, a excepción del cadmio cuya concentración es superior a $1,0 \mathrm{mg} / \mathrm{kg}$.

Los suelos son ácidos, con baja capacidad de carga iónica y baja saturación de bases; la profundidad efectiva es superficial a moderada con contenidos bajos de materia orgánica y altos contenidos de arena dispuestos sobre un valle de origen aluvial en posición de relieve ligeramente plano.

\section{AGRADECIMIENTOS}

Al Distrito de Riego el Juncal por el apoyo logístico, y la Universidad Surcolombiana por la dirección y asesoría en la investigación.

\section{REFERENCIAS}

Agencia para sustancias tóxicas y el registro de enfermedades (ATSDR). Consultado de: https://www.atsdr.cdc.gov/es/phs/es_phs5.html

Bernal-Fundora, A., Hernández-Jiménez, A., González-Cañizares, P. J., \& Cabrera-Rodríguez, A. 2019. Caracterización de dos tipos de suelos dedicados a la producción de plantas forrajeras. Cultivos Tropicales, 40(3), e05. Recuperado en 28 de abril de 2020, de 
http://scielo.sld.cu/scielo.php?script=sci_arttext\& pid=S0258-

$59362019000300005 \& \operatorname{lng}=e s \& \operatorname{lng}=e s$

Bighman J. y Bartels J. (1996). Organic matter Characterization. En: Methods of soil analysis. Part 3, Chemical methods (D. Sparks, Ed.), Soil Science Society of America, Book Series 5, Madison, WI, pp. 1011-1071

Boluda Hernández, R., Gimeno-García, E., \& Andreu Pérez, V. 2004. Impacto de la actividad agrícola sobre el aporte de metales pesados en suelos del Parque Natural de la Albufera de València. Dossiers Agraris, (7), 177-186.

Bravo, I., Arboleda, C. A., Martin, F. J. 2013. Efecto de la calidad de la materia orgánica asociada con el uso y manejo de suelos en la retención de cadmio, en sistemas altoandinos de Colombia. Ciencias del suelo. Acta agronómica. 63(2). 164-174p.

Brizuela, J. \& Jiménez, Y. 2012. Metodologías aplicadas para el establecimiento de los niveles de referencia para metales pesados en la evaluación de la contaminación en suelos. Avances en Química, 7 (2), 101-109.

Castro, A. 2015. Morphological, biochemical, molecular and ultrastructural changes induced by Cd toxicity in seedlings of Theobroma cacao L. Ecotoxicology and Environmental Safety, 174186.

Charrupi Riascos, N., \& Martínez Novoa, D. C. 2017. Estudio ambiental del cadmio y su relación con suelos destinados al cultivo de cacao en los departamentos de Arauca y Nariño. Retrieved from

https://ciencia.lasalle.edu.co/ing_ambiental_sanit aria/718

Dane - Fedearroz 2020. Boletín Técnico. Encuesta Nacional de Arroz mecanizado. 10 febrero de 2020. Bogotá. 26p.

Delince, W., Valdés Carmenate, R., López Morgado, O., Guridi Izquierdo, F., \& Balbín Arias,
M. I. 2015. Riesgo agroambiental por metales pesados en suelos con Cultivares de Oryza sativa L y Solanum tuberosum L. Revista Ciencias Técnicas Agropecuarias, 24(1), 44-50. Consultado de

http://scielo.sld.cu/scielo.php?script=sci_arttext\& pid=S2071-

00542015000100006\&lng=es\&tlng=es.

Díaz, E., Lenzi, L. y L. Donda (2018). Determinación de residuos de plaguicidas en suelos, aguas y granos en sistemas arroceros de Entre Ríos. Facultad de Ciencias Agropecuarias. Uner. 12p. Argentina.

Espinosa R., Adriana J. 2018. El agua, un reto para la salud pública La calidad del agua y las oportunidades para la vigilancia en Salud Ambiental Universidad Nacional de Colombia Facultad de Medicina Doctorado en Salud Pública Bogotá, Colombia. 194p.

Estévez J, Andrade M L, Marcet P, Montero M J. 2000. Fijación y movilidad de cadmio y zinc en tres tipos de suelos ácidos de Galicia, España. Ciencia del suelo, Vol. 18, №. 1, 2000, págs. 2835

http://www.suelos.org.ar/publicaciones/vol_18n1/ estevez_28-35.pdf

Fanfu Zeng, Wei Wei, Mansha Li, Ruixue Huang, Fei Yang, and Yanying Duan* 2015. Heavy Metal

Contamination in Rice-Producing Soils of Hunan Province, China and Potential Health Risks. Int J Environ Res Public Health. 2015 Dec; 12(12): 15584-15593.

Galán E. y Romero A. 2008. Contaminación de suelos por metales pesados. Universidad de Sevilla. $\mathrm{p}$ 48-68.

Gualdrón D., L. E. 2016. Evaluación de la calidad de agua de ríos de Colombia usando parámetros

Fisicoquímicos y biológicos, Revista Dinámica Ambiental, No 1, año 1. p 83-101.

Heinzen, J. \& Rodríguez, N. 2016. Procesos destructores para la salud vinculados a la 
manipulación de agroquímicos en trabajadores agrícolas de Young, Uruguay. Ciencia \& trabajo, 18(56), $117-$ 123. https://dx.doi.org/10.4067/S071824492016000200007

Organización de las Naciones Unidas para la Alimentación y la Agricultura (FAO) 1993. Joint FAO/WHO Food Standards programme, Codex Alimentarius Commission, 20th Session, Geneva June - July 1993.

Li, N. 2015. Concentration and transportation of heavy metals in vegetables and risk assessment of human exposure to bioaccessible heavy metals in soil near a waste-incinerator site, South China. Science of the Total Environment, 521-522, pp.144-151. Available at: DOI: http:// dx.doi.org/10.1016/j.scitotenv.2015.03.081.

Londoño-Franco L. F., P. T. Londoño-Muñoz, F.G. Muñoz-García (2016). Los riesgos de los metales pesados en la salud humana y animal. Biotecnología en el Sector Agropecuario y Agroindustrial Vol. 14 No. 2 (145-153).

McBride, M.B. 1995. Toxic metal accumulation from agricultural use of sludge: Are USEPA regulations protective? J. Environ. Qual. 24: 5-18

Mahecha-Pulido, Juan D., Trujillo-González, Juan M., \& Torres-Mora, Marco A. 2017. Análisis de estudios en metales pesados en zonas agrícolas de Colombia. ORINOQUIA, 21 (Suppl. 1), 83-93. http://www.scielo.org.co/pdf/rori/v21s1/01213709-rori-21-s1-00083.pdf

Munsell soil color charts. 1994. Little Britain Road. New Windsor. Agriculture Handbook.

Nicholson, F. A; Smith, S.R; Alloway, B.J; CarltonSmith, C; Chambers, B.J. 2003. An inventory of heavy metals inputs to agricultural soil in England and Wales. Science of the Total Environment. p. 205219.
Olivares Rieumont S., Damarys García Céspedes, Lázaro Lima Cazorla, Israel Saborit Sánchez, Axel Llizo Casals y Pedro Pérez Álvares. 2013. Niveles de cadmio, plomo, cobre y zinc en hortalizas cultivadas en una zona altamente urbanizada de la ciudad de la Habana, Cuba. Rev. Int. Contam. Ambient vol.29 no.4 México nov. 2013

Reyes Y. C., I. Vergara, O. E. Torres, M. Díaz, E. E. González 2016. Contaminación por metales pesados: implicaciones en salud, ambiente y seguridad alimentaria Revista Ingeniería, Investigación y Desarrollo, Vol. 16 No 2, JulioDiciembre 2016, pp. 66-77, Sogamoso-Boyacá. Colombia ISSN Impreso 1900-771X, ISSN Online 2422-4324

Rueda S., G., Rodríguez J. A., Madriñan R. 2011. Metodologías para establecer valores de referencia de metales pesados en suelos agrícolas: Perspectivas para Colombia. ACTA AGRONÓMICA. 60 (3) 2011, p 203-218.

Sierra, C. 2019. El Manganeso, el suelo y las plantas. Santiago de Chile. Consultado en: https://www.elmercurio.com/campo/noticias/anali sis/2016/03/09/el-manganeso-el-suelo-y-lasplantas.aspx

Tobón-Marulanda F. A., L. A. López-Giraldo y R. E. Paniagua-Suárez 2010. Contaminación del agua por plaguicidas en un área de Antioquia. Rev. Salud pública. 12 (2): 300-307.

Torrente, A. 2018. Impactos generados al ambiente y la salud humana por el uso de agroquímicos implementados en el cultivo de arroz (oryza sativa). Universidad Surcolombiana, Neiva.

Torrente, A. 2012. Estructuración del pedolarium del departamento del Huila con fines de exhibición, capacitación y transferencia de tecnología en suelos. Caracterización física, química y mineralógica de suelos. Universidad Surcolombiana. Neiva. 70p.

Trujillo, F. E. 2005. Impactos del plomo sobre los cultivos de arroz en Ibagué - Tolima, Finca la Argentina. Universidad de los Andes, Facultad de 
Ingeniería. Bogotá. 48p.

https://repositorio.uniandes.edu.co/bitstream/hand le/1992/22684/u270964.pdf?sequence=1\&isAllo wed $=\mathrm{y}$

Velásquez A. J. A. 2017. Contaminación de suelos y aguas por hidrocarburos en Colombia. Análisis de la fitorremediación como estrategia biotecnológica de recuperación. https://hemeroteca.unad.edu.co/index.php/riaa/arti cle/view/1846/2065

Yuan G. L., Sun T.H., Han P., Li J., Lang X.X. 2014. Source identification and ecological risk assessment of heavy metals in topsoil using environmental geochemical mapping: Typical urban renewal area in Beijing, China. Journal of Geochemical Exploration, 136, pp 40-47. DOI: http://dx.doi. org/10.1016/j.gexplo.2013.10.002 